(Aus dem physiologischen Institut der tierärztlichen Hochschule in Wien.)

\title{
Über \\ die Anpassung an einseitigen Vagusverlust bei Hund und Katze.
}

Von

Tierarzt Rudolf Petioky.

(Mit 2 Textfiguren.)

\section{Problemstellung und einleitende Versuchsergebnisse.}

Einseitige Ausschaltung des N. vagus am Halse unterhalb des N. laryngeus superior ist bei Hund und Katze zunächst in der Regel von keinen oder wenig merklichen Folgen für die Herztätigkeit und Atmung begleitet. Doch treten solche bald deutlicher hervor, um rasch ein Maximum zu erreichen, von dem aus ein allmäblicher Rückgang der Veränderungen erfolgt. Derselbe ist nicht an eine Regeneration bezw. Restitution des durchtrennten N. vagus geknüpft, sondern erfolgt auch, wenn eine solche nicht stattfindet. Es muss demnach ein gewisses Vikariieren beider Vagi eintreten, welches im Laufe der Zeit immer vollkommener wird. Als Grundlage für diese Erscheinung hat A. v. Tschermak ${ }^{1}$ ) die Tatsache erwiesen, dass der Hemmungstonus, welchen der eine N. vagus auf das Herz bei den höheren Säugetieren, speziell beim Hunde, ausübt, andauernd seitens des anderen Vagus beeinträchtigt wird. Es besteht also diesbezüglich eine antagonistische Wechselwirkung beider Herzvagi, so dass Hypotonie oder künstliche Ausschaltung der einen Leitung durch konsekutive Hypertonie der anderen kompensiert werden kann. Allerdings mag die letztere im Laufe der Zeit noch reaktiv verstärkt werden. Diese von ihm prinzipiell auf alle

1) A. v. Tschermak, Űber das Vikariieren der beiden Herzvagi. Monatsschrift f. Psychiatrie u. Neurol. Bd. 26, Ergänzungsband (Festschrift für Flechsig) S. 312--335. 1910. 
paarig tonisch-innervierten Gebilde ausgedehnte Vorstellung des Vikariierens auf Grund von antagonistischer Wechselwirkung hat A. v. Tschermak ${ }^{1}$ ) auf die Beobachtung gegründet, dasss bei Tieren mit Vagustonus temporäre oder dauernde Ausschaltung des einen Vagus zu einem Ansteigen der Erregbarkeit des anderen Vagus bzw. des teilweise gemeinsamen autonomen Endsystems beider Vagi führt.

Zudem ergab sich, dass sich der efferente Tonus des $\mathrm{N}$. vagus bioelektrisch im Sinne einer dauernden Degradation oder Depression des Längsquerschnittstromes äussert, indem Abtrennung des zentralen Vagusstumpfes von seiner medullaren Tonisierungsstätte ein Ansteigen des Stromes zur Folge hatte ${ }^{2}$ ). Da dieser Effekt eintrat, gleichgültig, ob der zweite Vagus erhalten oder zuvor durchtrennt war, erscheint es bereits erwiesen, dass der efferente Vagustonus, soweit er sich bioelektrisch äussert, nicht durch afferente Vagusfasern vermittelt ist, also nicht vago- bzw. autoreflektorischen Ursprunges ist.

Neben der antagonistischen Wechselwirkung der beiden efferenten Leitungen ist aus den Versuchen von J. Dog i el und E. Grahe ${ }^{3}$ ) auch auf einen reflektorischen, afferent-efferenten Hemmungseinfluss des einen Herzvagus auf den anderen zu schliessen. Bezüglich dieser Vorstellungen sei übrigens auf A. v. Tschermak's Darstellung (a. a. 0. 1910) verwiesen. Der letztere Autor behandelte bereits auch die Frage, ob der Herzvagustonus afferentreflektorischen oder rein efferent-automatischen Ursprungs sei.

Einen gewichtigen Beitrag zu diesem Probleme könnten, in Ergänzung zu A.v. Tschermak's bioelektrischen Untersuchungen, Beobachtungen über das Verhalten des kardialen Hemmungstonus nach Regeneration des $\mathrm{N}$. vagus abgeben, vorausgesetzt, dass es

1) Vgl. dessen die Lehre von der tonischen Innervation begründende Arbeiten: Studien über tonische Innervation. I. Pflüger's Arch. Bd. 119 S. 165-226. 1907. Studien über tonische Innervation. II. Pflüger's Arch. Bd. 136 S. 692-711. 1911. Über den Begriff der tonischen Innervation. Folia neurobiologica Bd. 1 S. 30-37. 1907. Über tonische und trophische Innervation. Folia neurobiologica Bd. 3 S. 676-694. 1909. Physiologische Untersuchnngen am embryonalen Fischherzen. Sitzungsber. d. Wiener Akad. d. Wissensch. Abt. III Bd. 118 S. 1-99. 1909.

2) A. v. Tschermak, Über bioelektrische Äusserung des Vagustonus. Pflüger's Arch. Bd. 136 S. 692-711. 1912.

3) J. Dogiel und E. Grahe, Über die Wechselwirkung der Nervi vagi auf das Herz. Du Bois' Arch. f. Physiol. 1895 S. 390-398. 
Über die Anpassung an einseitigen Vagusverlust bei Hund and Katze. 511 gelänge, den afferenten, sensiblen Anteil des Vagus dauernd ausgeschaltet $\mathrm{zu}$ halten bei Wiederherstellung des efferenten Anteiles. Würde längere Zeit nach einer dazu geeigneten Operation durch einen neuerlichen Eingriff der zweite früher intakt gelassene N. vagus durchtrennt und hierauf an dem regenerierten efferenten Anteil des anderen N. vagus noch ein kardialer Hemmungstonus nachzuweisen sein, so wäre damit sein efferent-automatischer Ursprung zwar noch nicht einwandfrei erwiesen, wohl aber wären - in Bestätiguing und Ergänzung der bioelektrischen Ergebnisse - die afferenten Leitungen im Vagus selbst als an der Tonuserzeugung unbeteiligt anzusehen, ein vago- bzw. autoreflektorischer Ursprung des efferenten Vagustonus ausgeschlossen.

Im Hinblick auf dieses Ziel machte Prof. A. v. Tschermak den Vorschlag, den einen N. vagus mit Einschluss seiner sympathischen Hülle in der Höhe des Foramen jugulare zentral vom Ganglion jugulare zu durchtrennen und unter Kontakt beider Stümpfe der Regeneration zu überlassen. Dabei ist im Prinzip zwar eine Restitution der efferenten, von ihren Zellhauptteilen oder Perikaryen abgetrennten Leitungen zu erwarten, nicht aber eine solche der afferenten, da die Spinalganglienzellen und ihre Homologa erfahrungsgemäss zwar den peripheren, nicht aber den zentralen Fortsatz zu regenerieren vermögen ${ }^{\mathbf{1}}$ ).

Die Durchführung dieses Planes erwies sich nun allerdings beim Hunde, als dem typisehen Versuchstiere für Studien über Vagustonus, schon dadurch hochoradig erschwert, dass das Ganglion jugulare zentral über dem Foramen jugulare nicht scharf abgegrenzt ist, sondern sich relativ boch hinauf als sogenanntes Ganglion nodosum fortsetzt, in welchem offenbar noch zahlreiche bipolare Ganglienzellen für afferente Vagusleitungen gelegen sind. Es liegt also die Gefahr nahe, dass nicht alle afferenten Vagusfasern zentral von ihren Ganglienzellen durchtrennt werden und daher die peripher davon durchtrennten ebenso regeneriert werden wie sämtliche efferente. In einem solchen Falle wird sich der regenerierte Vagus nebenbei

1) Über die verschiedene biologische Wertigkeit des zentralen und des peripheren Fortsatzes vgl. speziell G. Köster, Zur Physiologie der Spinalganglien und der trophischen Nerven sowie zur Pathogenese der Tabes dorsalis. Leipzig 1904. - Siehe auch A. Bethe, Allgemeine Anatomie und Physiologie des Nervensystems S. 182-233. Leipzig 1903. 
als sensibel erweisen, während ihm bei vollem Gelingen des Versuchsplanes Sensibilität völlig fehlen sollte, soweit nicht eine solche vorgetäuscht wäre durch zufällige Anlagerung fremder sensibler Leitungen, die bei der Operation mit durchtrennt wurden.

Solche Experimente zentraler Durchtrennung des rechtsseitigen N. vagus (mit Einschluss seiner sympathischen Hülle) wurden von mir unter aseptischen Kautelen an elf Hunden und zwei Katzen (vgl. Tabelle S. 513) durchgeführt, welche über 1 Jahr alt waren und, nach der relativ langsamen und speziell beim Hunde typisch wechselnden Schlagfrequenz $\mathrm{zu}$ schliessen, einen ausgebildeten Vagustonus besassen. Die Durchtrennung des Nervenstammes wurde möglichst weit zentral durch Scherensehlag vorgenommen und das Ganglion nodosum, möglichst weit hinauf durch das Foramen jugulare vordringend, entweder mechanisch durch Kneipen mit einer scharfgerieften Pinzette oder galvanokaustisch mittels einer besonders konstruierten Pinzette zerstört.

Nach längerer Zeit, bzw. nach 2-5 Monaten bei Hunden, 3-4 Monaten bei Katze (vgl. Tabelle S. 513), wurde im Morphium-Chloroformnarkose der durchtrennte Vagus am Halse aufgesucht und durch faradische Reizung mittels Schlitteninduktoriums (mit einem grossen Grenet'schen Tauchelement) in Kontinuität sowie nach Durchschneidung auf Regeneration bzw. Wiederherstellung der Hemmungswirkung auf das Herz geprüft.

Zur Reizung wurden Röhrenelektroden bzw. Platindrähte ver wendet, welche quer, halbkreisförmig gebogen in einer $3 \mathrm{~cm}$ langen, längsgeschlitzten Röhre aus Hartgummi lagen. Der Nerv war durch einen länglichen, den Schlitz schliessenden Wattestreifen in der Röhre fixiert und gedeckt. Unter Röhre und Nerv war noch zur Isolierung gegen Gewebsflüssigkeit und Blut ein Streifen wasserdichten Stoffes eingeschoben. Der Blutdruck wurde mittels Quecksilbermanometers am $\mathbf{L} \mathbf{u} \mathbf{d}$ w i $g$ ' schen Kymographion mit fortlaufendem Papier und Tintenschreibung registriert, ebenso die Atmung durch einen seitenständig an die Trachealkanüle angeschlossenen $\mathrm{M}$ areyschen Tambur; auch die 5-10 Sekunden betragende Reizdauer und die Zeit wurden markiert.

Bezüglich der Wirkung auf das Herz war das Ergebnis nur in drei Füllen ein positives, doch meist flüchtiges, und zwar bei Hund Nr. 3 (Schwelle bei $8 \mathrm{~cm}$, Effekt deutlicher bei $6 \mathrm{~cm} \mathrm{R.-A.),} \mathrm{am}$ besten bei Hund $\mathrm{Nr} .10$ (Schwelle bei $13-14 \mathrm{~cm}$, deutliche Senkung 
Über die Anpassung an einseitigen Vagusverlust bei Hund und Katze. 513

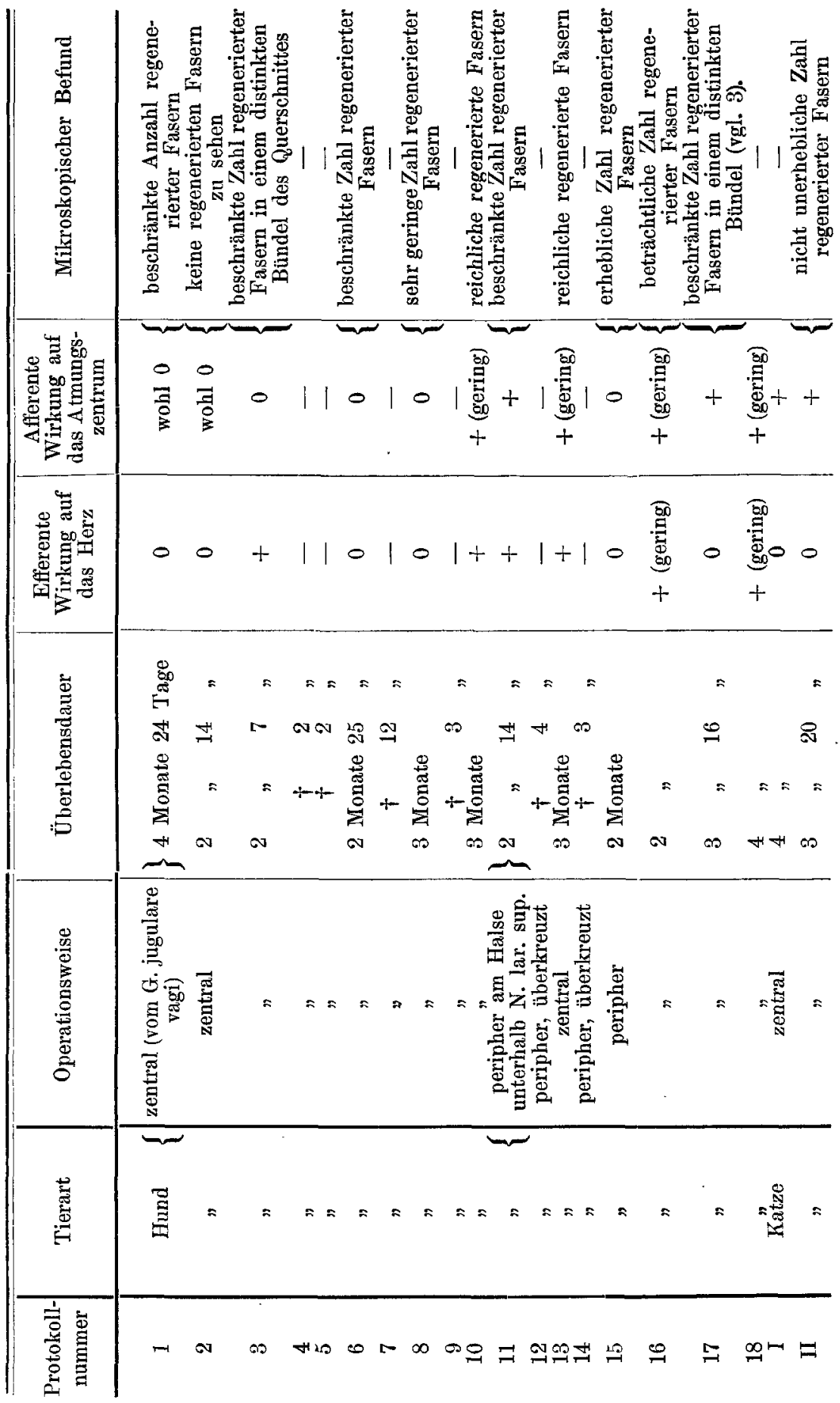


bei $12 \mathrm{~cm}$, Stillstand bei $10 \mathrm{~cm} \mathrm{R.-A.)} \mathrm{und} \mathrm{bei} \mathrm{Hund} \mathrm{Nr.} 13$ (Schwelle für Pulsverlangsamung und Drucksenkung bei $10 \mathrm{~cm}$, deutlicher und 1 Sekunde dauernder Stillstand bei $0 \mathrm{~cm}$ R.-A.). Man vergleiche damit die Reizungsergebnisse am normalen linksseitigen Vagus in Nr. 3 (bei Reizung des peripheren Stumpfes Schwelle $10 \mathrm{~cm}$ ), in $\mathrm{Nr} .10$ (bei Reizung in continuo Schwelle bei $15 \mathrm{~cm}$ starke Verlangsamung, doch kein Stillstand bei $0 \mathrm{~cm}$; bei Reizung des peripheren Stumpfes Schwelle bei $16 \mathrm{~cm}$, Stillstand bei $14 \mathrm{~cm}$ ), in Nr. 13 (bei Reizung in continuo Schwelle bei $22 \mathrm{~cm}$, Stillstand bei $12 \mathrm{~cm}$ ).

Ein völliges Fehlen der Sensibilität an dem zưr Regeneration bestimmten rechtsseitigen Vagus wurle in drei Versuchen (Hund Nr. 3, 6, 8) konstatiert, davon in zwei Fällen (Versuch Nr. 3 positiv) allerdings bei gleichzeitigem Fehlen einer Wiederherstellung der Wirkung auf das Herz. Während bei zwei Hunden (Hund Nr. 1 und 2) nur bei hohen Reizstärken (R.-A. 6 oder $4-0 \mathrm{~cm}$ ) reflektorische Unruhe und Abwehrbewegungen eintraten - ein Effekt, der auch auf Stromschleifen bezogen werden konnte -, erhielt ich bei zwei Hunden (Nr. 10 und 13, wenn auch in geringem Grade) und zwei Katzen schon bei Rollenabständen zwischen 10 und $15 \mathrm{~cm}$ vom Vagus in continuo bzw. nach vollzogener Durchtrennung von dem zentralen Stumpfe aus reflektorische Reaktionen, speziell Steigerung des Blutdruckes und Veränderung der Atmung (in Nr. 10 erst bei $5 \mathrm{~cm}$ R.-A. Stillstand). Bei Katze Nr. II waren solche Wirkungen auch nach Durchtrennung des rechtsseitigen Vagus vom peripheren Stumpfe her zu erhalten, demnach durch Neurotisation seitens anderweitiger mitdurchtrennter, dann regenerierter sensibler Nervenzweige vermittelt. In den erstgenannten Fällen war augenscheinlich eine gewisse Wiederherstellung von Sensibilität durch Regeneration afferenter, noch peripher von ihren Ganglienzellen durchtrennter Vagusfasern eingetreten - neben einer gleichzeitigen Regeneration von efferenten Herzhemmungsfasern: bei Hund Nr. 10 und 13 (bei Nr. 3 allein von efferenten).

Nach meinen Versuchen tritt jedenfalls eine erfolgreiche Regeneration der Vagusfasern, speziell der efferenten Herzhemmung vermittelnden, nach Durchtrennung an der Schädelbasis nicht leicht und nicht rasch ein. Ähnliches gilt bezüglich der Herzfasern auch nach Durehtrennung und Zusammennähen des Vagus am Halse, wäbrend sich die sensiblen Leitungen diesfalls leichter zu restituieren 
Über die Anpassung an einseitigen Vagusverlust bei Hund und Katze. 515

scheinen. In fünf bezüglichen Versuchen (Hund Nr. 11, 15, 16, 17, 18) wurde nach einigen Monaten nur in drei Fällen (Nr. 11, 16, 18) eine schwache, flüchtige Hemmungswirkung (bei $8,8,15 \mathrm{~cm}$ R.-A.) erhalten; selbst bei stärkster Reizung war keine erhebliche Verlangsamung oder gar Stillstand zu erzielen. Auch die sensiblen Leitungen erwiesen sich als nur unvollkommen regeneriert (geprüft in der reflektorischen Wirkung künstlicher Reizung auf die Atmung). Auch liess der nach Durchtrennung an der Schädelbasis oder am Halse der Regeneration überlassene Vagus keinen tonischen Einfluss auf das Herz erkennen, so dass seine Durchtrennung, im Gegensatz zu jener des normalen Vagus, die Pulsfrequenz und konsekutiv den Blutdruck nicht änderte. Auch zeigte sie keinen Einfluss auf die Reizbarkeit des anderen Vagus; es hatte sich also die normale beeinträchtigende Wecbselbeziehung der Herzvagi nicht wieder hergestellt.

Hingegen war in vier Fällen (Hund Nr. 11, 16, 17, 18) eine merkliche Regeneration der sensiblen Fasern eingetreten, so dass bei 8, 6, 12, $12 \mathrm{~cm}$ R.-A. (mit $17 \mathrm{~cm}$ als Schwelle) deutliche Wirkung auf die Atmung beobachtet wurde. - Endlich wurden in zwei Fällen (Hund Nr. 12 und 14) die beiden Vagi aus den längsgeschlitzten Sympathicusscheiden hervorgeholt, der eine höher, der andere tiefer unten zur Hälfte quereingeschnitten, die durchtrennten Fasern eine Strecke von ca. $5 \mathrm{~cm}$ weit nach unten bzw. nach oben von den undurchtrennten abgelöst und zwischen beiden Seiten durch. Naht eine Anastomose über der Trachea hergestellt. Es war beabsichtigt, bei längerem Üherleben der Tiere später den einen Vagus vollkommen zu durehtrenuen und so beide Seiten von demselben Vagus versorgen zu lassen. Nach der Operation bestand beiderseitiges Ektropium der Lider, Vorfall des dritten Augenlides, Miosis (scheinbarer Pupillendurchmesser $4 \mathrm{~mm}$ ), Schlingbeschwerden und Erbrechen. Nach 3 Tagen erfolgte in beiden Fällen der Tod (Hyperämie der Eingeweide, Lungenödem, Vagusherz, Inanition).

Die mikroskopische Untersuchung des der Regeneration überlassenen Vagus ergab das im letzten Stabe der Tabelle S. 511 detaillierte Resultat: eine gewisse, zum Teil allerdings recht beschränkte Regeneration von Nervenfasern. Die regenerierten Fasern waren - bei Untersuchung nach der Methode von MarchiAlgheri (Behandlung mit Müller'seher Flüssigkeit + Osmiumsäure) - durch hellgelbbraune Färbung und eine gewisse Körnung Pflüger's Archiv für Physiologie. Bd. 152. 
ihrer Markscheide kenntlich und kontrastierten scharf gegen die ungefärbt gebliebenen degenerierten Fasern, welche mehrfach schwarzgefürbte, häufig stabförmige Markschollen aufwiesen. Nicht immer war bei Vorhandensein regenerierter Fasern ein positiver Reizerfolg beobachtet worden, also eine funktionelle Restitution zu erschliessen. Wohl aber zeigten die im Reizversuch deutlich positiv befundenen Hunde Nr. 10 und 13 (weniger Hund Nr. 3) eine erhebliche Menge regenerierter Fasern in ihrem Vagus.

Bei dieser Sachlage (bloss drei Fälle von partieller Regeneration der efferenten Herzvaguszweige, davon nur einer ohne Regeneration der afferenten Vagusleitungen) war eine Antwort auf die eingangs gestellte Frage nach der Herkunft des Vagustonus nicht zu gewinnen. Dieselbe muss weiteren, vielleicht doch erfolgreichen Versuchen - etwa hei längerer Dauer oder an geeigneteren Tieren überlassen bleiben.

Ich musste mich daher damit begnügen, in den bisher erwähnten Versuchen den Verlauf der Anpassung an einseitigen Vagusverlust bei Hund und Katze zu verfolgen und zudem die Reizbarkeit des durchtrennten Vagus zu studieren.

\section{Verlauf der Anpassung an einseitigen Vagusverlust.}

Der Verlauf der Alteration, welche die Puls- und die Atmungsfrequenz infolge Durchtrennung des einen Vagus samt seiner sympathischen Hülle erfahren, sei durch die nachstehenden Kurven illustriert (Fig. 1 und 2). In denselben ist zunächst das Niveau vor der Operation, dann - nach Unterbrechung durch das Intervall des Operationstages - der Verlauf des Frequenzabfalles verzeichnet. Über die Art des Anstieges während der Operation und der folgenden 24 Stunden erscheint sonach keine Aussage gemacht. Der Abfall ist im allgemeinen wohl als ein stetiger anzusehen, indem deutliche neuerliche Anstiege kaum zu verzeichnen sind. Mehrfach nähert sich der Ahfall der Pulsfrequenz einer geraden Linie, während jener der Atmungsfrequenz anfangs rascher, später langsamer zu erfolgen scheint.

Die Steigerung der Pulsfrequenz beträgt bei Hunden nach 24 Stunden durchschnittlich 149 (139-159) gegen 100 (96-106), also annähernd $50 \%$; bei Katzen nach 24 Stunden 230 oder mehr als 240 gegen 120 vor der Operation, also bis $100 \%$, ja darüber. 
Über die Anpassung an einseitigen Vagusverlust be: Hund und Katze. 517

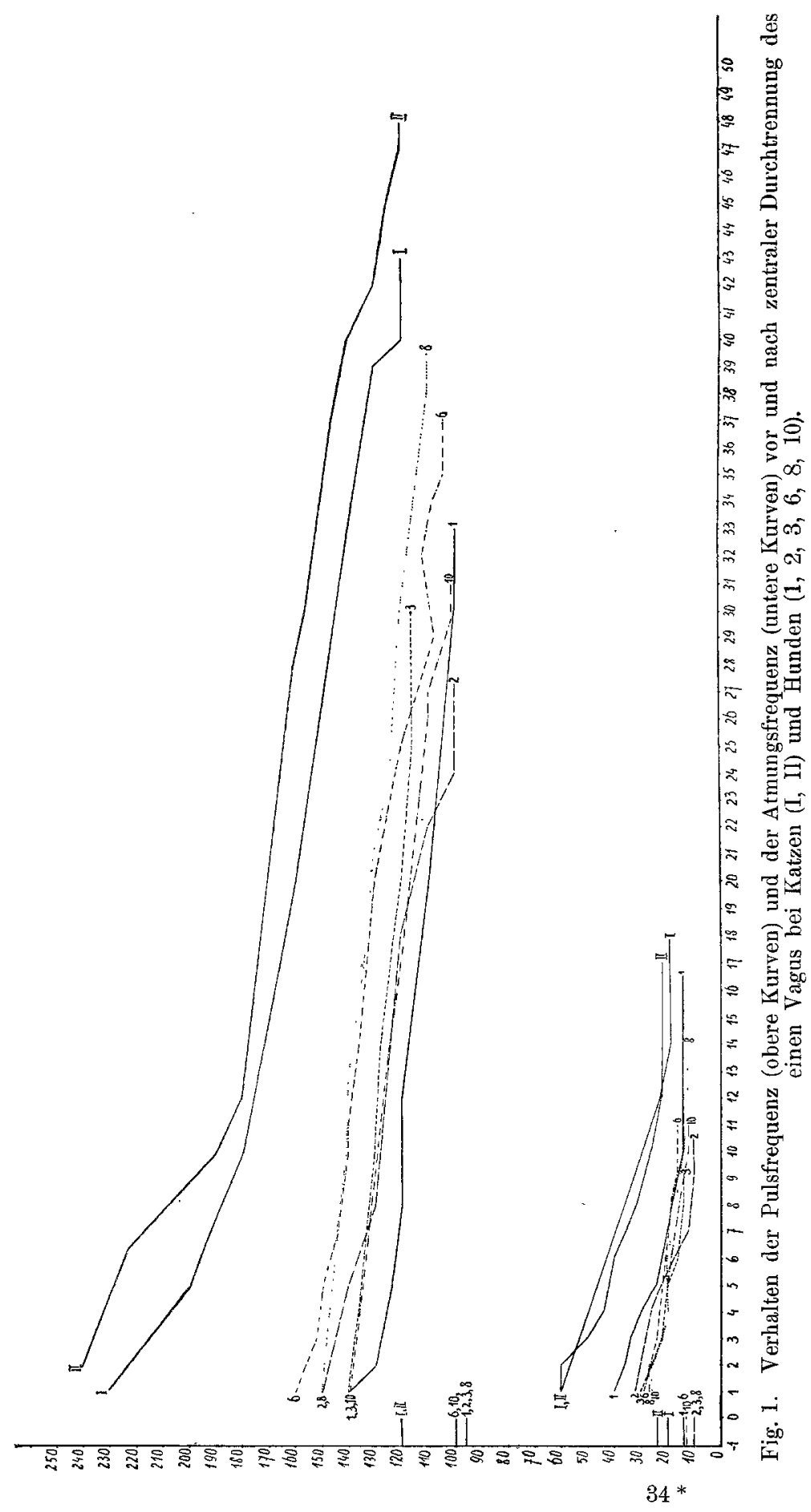




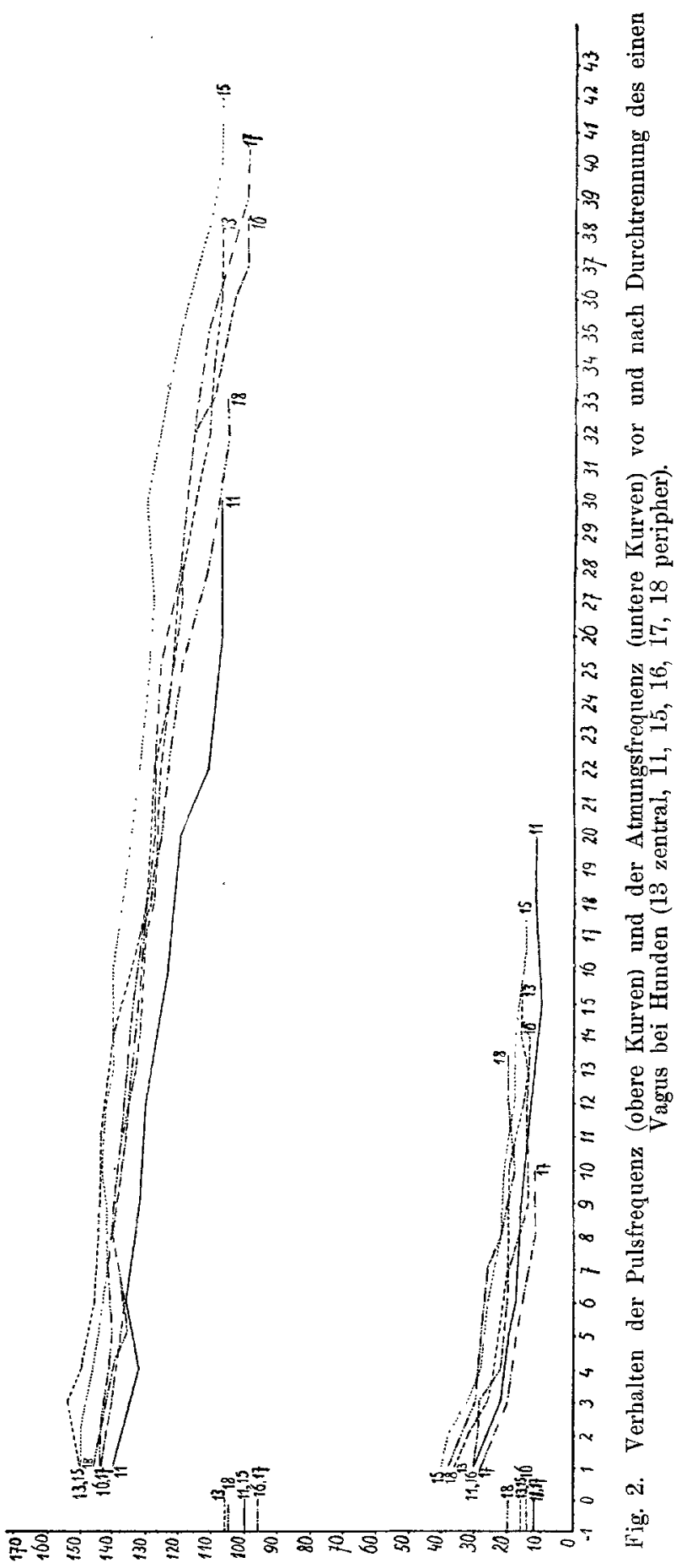


Über die Anpassung an einseitigen Vagusverlust bei Hund und Katze. 519

Eine Rückkehr zum Ausgangsniveau wird bei Hunden und Katzen nicht vor 4 Wochen bzw. bei Hunden etwa nach $32-42$, bei Katzen nach 40-47 Tagen erreicht, ohne dass der Schluss erlaubt wäre, dass die Pulsfrequenz in jedem Einzelfalle schliesslich auf den vor der Operation bestehenden Durchschnittswert sicher herabgehen würde. Die Steigerung der Pulsfrequenz ist unabhängig davon, ob die Durchtrennung des einen Vagus an der Schädelbasis oder am Halse vorgenommen wird. Ihr Eintreten beweist die Unvollkommenheit des Vikariierens des zweiten Vagus nach einem Intervall von 24 Stunden und später bei einseitiger Vagotomie. Offenbar reicht die nach Ausschaltung des einen Herzvagus erfolgende Steigerung der Erregbarkeit bzw. der tonisehen Beeinflussbarkeit des intrakardialen Hemmungsapparates (nach A. v. Tschermak) nicht aus, um, speziell auf die Dauer, den efferenten tonischen Einfluss des anderen Herzvagus bis zur Höhe des bisher von beiden Nerven vereint ausgeübten Effektes anwachsen zu lassen. Das Verbalten binnen der ersten 24 Stunden, welches allerdings durch äussere Einflüsse koupliziert ist, bedürfte noch der Untersucbung. Auch ubber den Morlus der allmählich eintretenden Kompensation, die wohl in der Regel schliesslich eine vollkommene wirl, lässt sich heute noch nichts Näheres aussagen. Nur das ist zu betonen, dass dieser Vorgang unabbängig erscheint von einer durch den Reizversuch und den histologischen Befund erwiesenen Regeneration des durchtrennten Vagus.

Die Steigerung der Atmungsfrequenz beträgt bei Hunden nach 24 Stunden durchschnittlich $32(25-40)$ gegen $15(10-20)$, also etwas uber $50 \%$; bei Katzen nach 24 Stunden 60 gegen 22 (20-24) vor der Operation, was nahezu eine Verdreifachung der normalen Frequenz bedeutet. Die Rückkehr zur Norm erfolgt erheblich rascher als bezüglich der Pulsfrequenz, und zwar binnen 10-14 Tagen Der Grad der Steigerung ist anch in diesem Falle unabbängig von der Durchtrennungsstelle. Die beobachtete Zunahme der Atmungsfrequenz nach einseitiger Vagotomie steht im Gegensatze zu der bekanntlich nach doppelseitiger Vagusdurchtrennung erfolgenden Verlangsamung und Vertiefung der Atmung - eine Veränderung, die auf Wegfall eines afferenten Beschleunigungstonus der Vagi bezogen wird. Der Versuch, heute schon eine Erklärung für die bezeichnete gegensinnige Folge einseitiger Vagotomie aufzustellen, sei unterlassen. Nur sei bemerkt, dass es wenig Wahrscheinlichkeit für sich hätte, an eine abklingende Dauerreizung des zentralen Vagusstumpfes durch 
den Wundheilungsprozess oder durch eine sogenannt aufsteigende Degeneration der zum medullaren Atmungszentrum emporziehenden Vagusfasern zu denken.

Ausfallserscheinungen im Gebiete des Kopfsympathicus (Ektropium, speziell des unteren Lides, Vorfall des dritten Augenlides bis zu einem Drittel der Hornhaut, Verengerung der Pupille, z. B. $3 \mathrm{~mm}$ gegen $6-8 \mathrm{~mm}$ scheinbaren Durchmessers auf der gesunden Seite beim Hunde, $3 \mathrm{~mm}$ gegen $8-10 \mathrm{~mm}$ bei der Katze bei starker Tagesbeleuchtung) wurden nur nach Durchtrennung des einen Vagus an der Schädelbasis sowie nach Halbdurehtrennung beider Vagi am Halse (Fall Nr. 12 und 14) beobachtet. In einem Falle von einseitiger basaler Durchtrennung des Vagosympathicus an einem Hunde (Nr. 3) wurden jegliche sympathische Ausfallserscheinungen vermisst, trotz vollständiger Durchtrennung und charakteristischer Beschleunigung von Puls- und Atmungsfrequenz. Weiters wurde in der Regel eine erhebliche Steigerung der Tränensekretion auf der operierten Seite beobachtet. Das Fehlen von Ausfallserscheinungen nach einseitiger Vagotomie am Halse ist wohl auf eine "subsidiäre A u tomatie" der Neuronen des oberen Halsganglions zu beziehen. Für das Zurück* gehen der Ausfallserscheinungen in jenen Fällen, in welchen oberbalb des Gangl. cervic. sup. durchtrennt wurde, also dessen postganglionäre Fasern ausgeschaltet wurden, dürfte - von der Möglichkeit einer Regeneration abgesehen - in erster Linie eine subsidiäre Automatie peripher gelegener Ganglienzellstationen oder ein Anwachsen des Eigentonus der zugehörigen glatten Muskeln selbst verantwortlich zu machen sein.

Der Rückgang des Sympathicusausfalles war binnen etwa 3 Wochen ein weitgehender; doch blieb das Ektropium bzw. besonders der Vorfall des dritten Lides sowie auch die Pupillenverengung auf der operierten Seite angedeutet.

\section{Vergleich der Reizbarkeit des undurchtrennten und des am Halse durehtrennten Vagus.}

An den rechterseits, sei es basal, sei es am Halse, vagotomierten Tieren wie auch an einem nicht operierten Hunde wurde ferner die Reizbarkeit des normalen $N$. vagus in Kontinuität wie nach der Durchschneidung geprüft. Im letzteren Falle wurden die Röhrenelektroden möglichst entfernt von der Schnittstelle angelegt, um eine eventuelle Beeinflussung der Reizbarkeit durch die Nähe des Quer- 
Über die Anpassung an einseitigen Vagusverlust bei Hund und Katze. 521

\begin{tabular}{|c|c|c|c|c|}
\hline 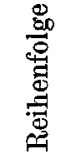 & Versuchstier & $\begin{array}{l}\text { Wirkung auf } \\
\text { Herz oder } \\
\text { Atmung }\end{array}$ & $\begin{array}{l}\text { A. Effekte bei Reizung } \\
\text { des normalen Vagus } \\
\text { in continuo }\end{array}$ & $\begin{array}{l}\text { B. Effekte bei Reizung } \\
\text { des normalen Vagus } \\
\text { nach Durchschneidung }\end{array}$ \\
\hline I. & $\begin{array}{l}\text { Hund Nr } 6 \text {, } \\
\text { rechts basal } \\
\text { vagotomiert }\end{array}$ & Herz & $\begin{array}{l}15 \mathrm{~cm} \mathrm{R.-A.} \mathrm{effektlos} \mathrm{(0)} \\
12 \mathrm{~cm} \text { R.-A. Pulsverlang- } \\
\text { samung }\end{array}$ & $\begin{array}{l}16 \mathrm{~cm} \mathrm{R} \cdot-\text { A. Pulsverlangs. } \\
15 \mathrm{~cm} \mathrm{R.-A.} \mathrm{Stillstand}\end{array}$ \\
\hline II. & $\begin{array}{l}\text { Hund Nr. } 8, \\
\text { rechts basal } \\
\text { vagotomiert }\end{array}$ & Herz & $\begin{array}{l}15 \mathrm{~cm} \mathrm{R.-A.} 0 \\
14 \mathrm{~cm} \text { R.-A. Stillstand }\end{array}$ & $\begin{array}{c}17-18 \mathrm{~cm} \mathrm{R} \cdot-\text { A. Schwelle } \\
16 \mathrm{~cm} \text { R.-A. Stillstand } \\
(4 \mathrm{~cm} \text { von der Schnittläche } \\
\text { entfernt })\end{array}$ \\
\hline III. & $\begin{array}{l}\text { Hund Nr. } 10, \\
\text { rechts basal } \\
\text { vagotomiert }\end{array}$ & Herz & $\begin{array}{l}15 \mathrm{~cm} \text { R.-A. Schwelle } \\
0 \text { cm R.-A. Pulsverlang- } \\
\text { samung (kein Stillstand) }\end{array}$ & $\begin{array}{l}16 \mathrm{~cm} \mathrm{R.-A.} \mathrm{Schwelle} \\
14 \mathrm{~cm} \mathrm{R.-A.} \mathrm{Stillstand} \\
\text { (maximal entfernt) }\end{array}$ \\
\hline IV. & $\begin{array}{l}\text { Hund Nr. 15, } \\
\text { rechts unter } \\
\text { N. laryng. } \\
\text { super. vagotom. }\end{array}$ & Herz & $\begin{array}{c}15-20 \mathrm{~cm} \text { R.-A. Schwelle } \\
8 \mathrm{~cm} \text { R.-A. Pulsverlang- } \\
\text { samung } \\
\text { (kein Stillstand) }\end{array}$ & $\begin{array}{c}15 \mathrm{~cm} \mathrm{R} \cdot-A . \text { Schwelle } \\
13-14 \mathrm{~cm} \mathrm{R.-A} \text {. Stillstand } \\
(3 \mathrm{~cm} \text { entfernt })\end{array}$ \\
\hline & & Atmung & $\begin{array}{l}20 \mathrm{~cm} \text { R.-A. Schwelle } \\
18 \mathrm{~cm} \text { R.-A. Stillstand }\end{array}$ & $\begin{array}{l}22 \mathrm{~cm} \mathrm{R} \text {. }-A . \text { Verlangsamung } \\
18-20 \mathrm{~cm} \text {. R.-A. Stillstand }\end{array}$ \\
\hline V. & $\begin{array}{l}\text { Hand Nr. 16, } \\
\text { rechts unter } \\
\text { N. laryng. }\end{array}$ & Herz & $\begin{array}{l}14 \mathrm{~cm} \mathrm{R.-A.} \mathrm{Schwelle} \\
12 \mathrm{~cm} \mathrm{R}-\bar{A} . \text { Stillstand }\end{array}$ & $\begin{array}{l}15 \mathrm{~cm} \text { R.-A. } 0 \\
14 \mathrm{~cm} \text { R.-A. Schwelle } \\
12 \mathrm{~cm} \text { R.-A. Stillstand }\end{array}$ \\
\hline & & Atmung & $\begin{array}{c}20 \mathrm{~cm} \text { R.-A. } 0 \\
18 \mathrm{~cm} \mathrm{R.-A.} \mathrm{Schwelle} \\
15 \mathrm{~cm} \text { R.-A. deutliche Ver- } \\
\text { langsamung } \\
12 \mathrm{~cm} \text { R.-A. Stilistand }\end{array}$ & $\begin{array}{c}20 \mathrm{~cm} \mathrm{R.-A.} 0 \\
18 \mathrm{~cm} \mathrm{R.-A.} \mathrm{Schweile} \\
-\end{array}$ \\
\hline VI. & $\begin{array}{l}\text { Hund Nr. 17, } \\
\text { rechts unter } \\
\text { N. laryng. } \\
\text { super. vagotom. }\end{array}$ & Herz & $\begin{array}{l}12 \mathrm{~cm} \mathrm{R.}-A .0 \\
11,5 \mathrm{~cm} \text { R.-A. Stillstand }\end{array}$ & $\begin{array}{c}12,8 \mathrm{~cm} \mathrm{R.-A.} 0 \\
12,6 \mathrm{~cm} \text { R.-A. Stillstand }\end{array}$ \\
\hline VII. & $\begin{array}{l}\text { Hund Nr. 18, } \\
\text { rechts unter }\end{array}$ & Herz & $\begin{array}{l}26 \mathrm{~cm} \mathrm{R}-\text { A. Verlangsamung } \\
20 \mathrm{~cm} \text { R.-A. Stillstand }\end{array}$ & $\begin{array}{l}27 \mathrm{~cm} \text { R.-A. Schwelle } \\
26 \mathrm{~cm} \text { R.-A. Stillstand }\end{array}$ \\
\hline & super. vagotom. & Atmung & $\begin{array}{l}45 \mathrm{~cm} \text { R.-A. Schwelle } \\
32 \mathrm{~cm} \text { R. }- \text { A. Stillstand }\end{array}$ & $45 \mathrm{~cm} \mathrm{R}$-A. Schwelle \\
\hline VIII. & $\begin{array}{l}\text { Hund } \mathrm{Nr} .19 \\
\text { normal }\end{array}$ & Herz, r. Vagus & $\begin{array}{l}15 \mathrm{~cm} \text { R.-A. Schwelle } \\
14 \mathrm{~cm} \mathrm{R.-A.} \mathrm{Stillstand}\end{array}$ & $\begin{array}{l}16,5 \mathrm{~cm} \mathrm{R.-A.} 0 \\
16 \mathrm{~cm} \mathrm{R.-A.} \mathrm{Stillstand}\end{array}$ \\
\hline & & Herz, l. Vagus & $\begin{array}{l}14,5 \mathrm{~cm} \mathrm{R} \text {-A. Schwelle } \\
14 \mathrm{~cm} \mathrm{R} \text {-A. Stillstand }\end{array}$ & $\begin{array}{l}14 \mathrm{~cm} \text { R. A. Schwelle } \\
13,8 \mathrm{~cm} \text { R.-A. Stillstand }\end{array}$ \\
\hline IX. & $\begin{array}{l}\text { Katze Nr. II, } \\
\text { rechts basal } \\
\text { vagotomiert }\end{array}$ & $\begin{array}{c}\text { A tmung, r. Vag. } \\
n .1 . " \% \\
\text { Herz }\end{array}$ & $\begin{array}{c}20 \mathrm{~cm} \text { R.-A. Schwelle } \\
20 \mathrm{~cm} \text { R.-A. Schwelle } \\
15 \mathrm{~cm} \text { R.-A. Pulsverlang- } \\
\text { samung } \\
13 \mathrm{~cm} \text { R.-A. kein Stillstand }\end{array}$ & $\begin{array}{l}22 \mathrm{~cm} \text { R.-A. Schwelle } \\
22 \mathrm{~cm} \text { R.-A. Schwelle } \\
16 \mathrm{~cm} \text { R.-A. Schwelle } \\
15 \mathrm{~cm} \text { R.-A. Stillstand }\end{array}$ \\
\hline
\end{tabular}


schnittes auszuschliessen. Besondere Versuche mit Verschiebung der Elektroden an den Querschnitt ergaben übrigens keine irgendwie nennenswerte lokale Verschiedenheit der Reizbarkeit. Zudem nimmt die später zu beschreibende Steigerung der Reizbarkeit nach der Durchtrennung anfangs mit der Zeit $z u$, während der Längsquerschnittstrom stetig absinkt, und zwar anfangs ziemlich rasch.

Die Versuche betrafen in erster Linie die Reizbarkeit der efferenten Herzvagusfasern; nur nebenbei wurde mitunter auch jene der afferenten, auf das medullare Atmungszentrum wirkenden Vagusfasern geprüft.

Über die einzelnen Versuche berichtet die vorstehende Tabelle (S. 521).

Es ergibt sich, dass Durehtrennung des Vagus, sei es basal, sei es am Halse, ein mässiges Ansteigen der faradischen Reizbarkeit seiner efferenten Hemmungsasern zur Folge hat, was sich an einem Sinken der Schwelle für Pulsverlangsamung und Blutdrucksenkung sowie des Reizwertes für Herzstillstand kundgibt. Es ist somit eine tonische Selbstbeeinträchtigung bzw. Selbstregulierung der tonischen Hemmungsfasern des einzelnen Vagus zu erschliessen. Hingegen lässt sich bezüglich der afferenten Atmungsfasern kein regulärer Einfluss der Durchtrennung feststellen.

Der obige positive Befund fügt sich als Ergänzung an die bereits eingangs erwähnte Beobachtung A. v. Tschermak's, dass Durchtrennung des einen Vagus ein mitunter recht erhebliches Ansteigen der Reizbarkeit des peripheren Stumpfes bzw. der efferenten Herzfasern des anderen Vagus, gleichzeitig auch ein Ansteigen des Längsquerschnittstromes des zentralen Stumpfes des anderen Vagus nach sich zieht.

Allerdings muss $e$ unentschieden bleiben, ob die tonische Selbstbeeinträchtigung des einzelnen Herzvagus ebenso durch eine efferente depressive Dauerwirkung auf den kardialen Hemmungsapparat zu erklären ist, wie die wechselseitige Beeinträchtigung beider Vagi nach A. v. Tschermak's Versuchen. Ebenso wie in letzterer Beziehung neben der efferenten Komponente noch eine afferentefferente oder reflektorische Komponente anzunehmen ist, und zwar auf Grund der Versuchsergebnisse von J. Dogiel und E. Grahe ${ }^{1}$ ),

1) J. Dogiel und E. Grabe, Du Bois' Arch. f. Anat. u. Physiol. 1895 S. 390-399. Vgl. die diesbezüglichen Ausführungen bei A. v. Tschermak, Monatsschr. f. Psychiatr. u. Nenrol. Bd. 16, Ergänzungsband (Festschrift für Flechsig) S. 312-335. 1910. 
Über die Anpassung an einseitigen Vagusverlust bei Hund und Katze. 523

könnte auch die aus meinen Beobachtungen zu erschliessende Selbstbeeinträchtigung des einzelnen Herzvagus afferent-efferenter oder reflektorischer Natur sein. Eine Entscheidung zwischen den beiden angedeuteten Möglichkeiten kann am normalen Vagus nicht getroffen werden; hingegen wäre sie möglich an einem rein efferent regenerierten Vagus, dessen Herstellung in den einleitenden Versuchen, allerdings im wesentlichen vergeblich, angestrebt worden war.

\section{Zusammenfassung.}

1. Es wurde die Herstellung eines rein efferenten N. vagus beim Hunde durch Regeneration nach Durchtrennung zentral vom G. jugulare vagi, inklusive G. nodosum, angestrebt, um die Frage der efferent-automatischen oder afferent-efferenten bzw. reflektorischen Natur des normalen Vagustonus zu entscheiden. Das beabsichtigte Ziel wurde nur teilweise und unvollkommen erreicht, so dass das bezeichnete Problem offen gelassen werden muss. Doch könnten analoge Versuche an einem grösseren, durch längere Zeit beobachteten Material und an geeigneteren Tieren von Erfolg sein.

2. Die Ausfallserscheinungen nach einseitiger Ausschaltung des $\mathrm{N}$. vagus und des Halssympathicus an der Schädelbasis gehen, auch bei Ausbleiben einer Regeneration bezw. Restitution, allmählich, anfangs rasch, dann immer langsamer zurück, so dass in $3-5$ Wochen die normale Puls- und Atmungsfrequenz wieder erreicht wird, wäbrend Spuren des Sympathicusausfalles bestehen bleiben. In einem Falle von basaler Vagotomie sowie in den Fällen von einseitiger Durchtrennung am Halse wurden Erscheinungen von Sympathicusausfall dauernd und vollständig vermisst.

3. Durchtrennung eines Vagus, sei es basal oder am Halse, hat ein mässiges Ansteigen der faradischen Reizbarkeit seiner efferenten Herzhemmungsfasern zur Folge. Aus diesem Verhalten ist eine tonische Selbstbeeinträchtigung der kardialen Hemmungsfunktion des einzelnen Vagus zu erschliessen. Ob diese Selbstregulierung rein efferenten oder reflektorischen Charakter besitzt, muss dahingestellt bleiben.

Zum Schlusse sei es mir gestattet, Herrn Prof. Dr. A. v. Tscher mak für seine mannigfaltigen Anregungen und tatkräftige Beibilfe bei meiner Arbeit meinen herzlichsten Dank auszusprechen. Desgleichen danke ich meinen Kollegen, den Herren Assistenten Tierarzt Hans Hitzker und Erich Goldschmidt für ihre freundliche Mithilfe aufs beste. 